\title{
Evaluation of the Sympathetic Skin Response in Men with Chronic Prostatitis: A Case-Control
} Study

This article was published in the following Dove Press journal: Research and Reports in Urology

\author{
Ali Eslahi iD ${ }^{1,2}$ \\ Hamidreza Farpour (iD) 2,3 \\ Azar Hosseini iD ${ }^{4}$ \\ Faisal Ahmed iD ${ }^{5}$ \\ Umayir Chowdhury iD ${ }^{4}$ \\ Hossein-Ali Nikbakht ${ }^{6}$ \\ 'Department of Urology, Shiraz \\ University of Medical Sciences, Shiraz, \\ Iran; ${ }^{2}$ Shiraz Geriatric Research Center, \\ Shiraz University of Medical Sciences, \\ Shiraz, Iran; ${ }^{3}$ Physical Medicine and \\ Rehabilitation Specialist, Bone and Joint \\ Diseases Research Center, Department \\ of Physical Medicine and Rehabilitation, \\ Shiraz University of Medical Sciences, \\ Shiraz, Iran; ${ }^{4}$ Student Research \\ Committee, Shiraz University of Medical \\ Sciences, Shiraz, Iran; ${ }^{5}$ Urology Research \\ Center, Al-Thora General Hospital, \\ Department of Urology, lbb University of \\ Medical Since, Ibb, Yemen; ${ }^{6}$ Social \\ Determinants of Health Research Center, \\ Health Research Institute, Babol \\ University of Medical Sciences, Babol, \\ Iran
}

Background: Etiological factors involved in chronic prostatitis (CP) type IIIb and chronic pelvic pain are not sufficiently understood; however, the nervous system has a significant role in the generation and maintenance of chronic pelvic pain. This study was designed to evaluate the sympathetic skin response (SSR) in men with CP type IIIb compared to normal population.

Patients and Methods: For two years, about 14 patients suffering from CP type IIIb according to NIH-CPSI and 26 healthy control men were enrolled in this study. SSR was performed in all the subjects with a standard method. Bilateral palmar and plantar latency and amplitude of SSR were recorded in response to the median and tibial nerve electrical stimulations. SSR is considered abnormal when the latency is prolonged, and the amplitude reduced.

Results: SSR latency in the left and right median nerve was significantly prolonged in the patient with $\mathrm{CP}$ type IIIb group compared to the control group ( $\mathrm{p}=0.039$ and 0.006 , respectively). Additionally, the amplitude was reduced in patients with CP type IIIb group compared to the control group in the right tibial nerve $(\mathrm{p}=0.017)$.

Conclusion: Sympathetic skin response may be a helpful diagnostic test for men with chronic prostatitis type IIIb. However, this observation needs to be validated in a large sample cohort study with long-term follow-up.

Keywords: chronic prostatitis, prostate, sympathetic skin response

\section{Introduction}

Male chronic pelvic pain is commonly diagnosed as chronic prostatitis (CP) type IIIb or non-inflammatory subtype. It has a severe negative impact on the patients' quality of life. ${ }^{1}$ Prostatitis-like symptoms, especially pelvic pain, occur with a prevalence of about $8 \% .^{2}$ Many different etiologies and mechanisms of pathogenesis of CP have been suggested in the past ten years, including a role for immunologic, infective, endocrine, and psychological factors. However, none of these hypotheses fully account for all recognized clinical and experimental findings. ${ }^{3}$

Recently, there has been a gradual recognition that the nervous system, particularly the ANS (autonomic nervous system), has a significant role in the generation and maintenance of chronic pelvic pain. ${ }^{4}$ ANS changes can be altered by SSR using a clinical neurophysiological equipment to evaluate the autonomic innervation of the skin. Electrodermal findings are usually symmetric between the extremities. Asymmetric SSRs are found when one limb is denervated or injured and in chronic
Correspondence: Faisal Ahmed Email fmaaa2006@yahoo.com 
pain disorders such as complex regional pain syndrome and electrical burn injury. ${ }^{5}$ The SSRs are a polysynaptic reflex generated by reflex activation of the sweat glands via cholinergic sudomotor sympathetic efferent fibers. ${ }^{6}$ It has been used to show the sympathetic nervous system derangement in a wide spectrum of diseases such as central and peripheral neuropathies, rheumatologic problems, spinal cord injury, Crohn's disease, and in burn injuries. ${ }^{7}$ A previous study showed that generalized ANS changes were observed in men with chronic pelvic pain syndrome (CPPS). These changes are reflected by altered cardiac responses to postural change and higher peripheral blood pressure measurements.

This study was designed to evaluate the sympathetic skin response in men with chronic prostatitis type IIIb as a diagnostic method compared to a normal population.

\section{Patients and Methods}

The sample included 26 healthy individuals and 14 patients with $\mathrm{CP}$ type IIIb who were diagnosed according to NIHCPSI by a urologist in the period between January 1, 2018 and December 30, 2019.

Subjects with CP type IIIb were recruited from the prostatitis clinic at the University of Shiraz, and control subjects without CP were recruited via advertisements. The ethics committee of Shiraz University of Medical Sciences with the ID: 17229 approved this study. Additionally, all participants provided written, informed consent to be enrolled in our study.

\section{Exclusion Criteria}

The exclusion criteria were recent urinary tract infection (UTI); postoperative pain; previous radiation therapy; and any urological intervention for the bladder, prostate, or kidney; urinary tract malignancies; a history of genitourinary tuberculosis; any known neurological abnormalities including spinal cord injury; skin grafts, severe scarring; amputation of one or more limbs; abnormal nerve conduction studies; any Co-morbidities such as cardiovascular disease, lung diseases, neurological diseases (such as neuropathies), Glandular diseases (such as diabetes or thyroid disorders), metabolic diseases, vascular collagen diseases (such as systemic lupus erythematosus, rheumatoid arthritis, and scleroderma), history of surgery on the upper or lower extremities. Overt psychiatric disease; and patient's age younger than 18 or older than 65 years.

\section{Inclusion Criteria}

The inclusion criterion was the patients diagnosed with $\mathrm{CP}$ type IIIb according to the National Institutes of Health criteria (NIH-CPSI). ${ }^{8}$ All patients reported genitourinary pain, which was localized at the lower back, lower abdomen, anus, penis, testis, perineum, with or without voiding symptoms (frequency, urgency, weak stream, nocturia), and ejaculation pain. The symptoms were recorded in the absence of uropathogenic bacteria (as detected by standard microbiologic methods), benign prostatic hyperplasia, tumors, or other identifiable causes such as known neurologic abnormalities or psychiatric diseases. The chronic pelvic pain had lasted for at least three months of the preceding six months at diagnosis. All the patients had received prior treatments consisting of antibiotics, antiinflammatories, plant extracts, alpha1-blockade, 5-alphareductase inhibitors, antidepressants, antimuscarinics, anxiolytics, and neuromodulation agents. Two weeks before the test, all the patients stopped any medications used to control their specific prostatic symptoms. Throughout the study, neither group received drugs that could influence the results, such as antibiotics, antihistamines, antidepressants, clonidine, ergotamine, betablockers, serotonergic drugs, oral contraceptives, or pain relievers.

\section{Sympathetic Skin Responses}

The study was conducted as standard conditions and in a semi-dark and quiet room. The patient's skin temperature was controlled and maintained above $32^{\circ} \mathrm{C}$ degrees by an electronic thermometer before and after the test. For the SSR test, the patient lay in a supine position on the bed, and the room temperatures were kept between $23^{\circ} \mathrm{C}$ and $26^{\circ} \mathrm{C}$ and the humidity between $30 \%$ and $35 \%$. ${ }^{9}$ The skin was cleansed and covered with electrolyte gel. SSR test was performed by circular shaped ( $6 \mathrm{~mm}$ disk electrodes) surface electrodes and an electromyography device; Medelec Synergy electromyography instrument (VIASYS Healthcare UK, manor way, old-working, Surrey, UK).

At first, we did a nerve conduction study to exclude the peripheral neuropathies; then, for the hand, to record the median nerve SSR, an active electrode was placed on the palmar surface, a reference electrode on the dorsal aspect of the hand, and a ground electrode on the forearm. The ipsilateral median nerve was stimulated. (Figure 1)

For the foot, to record the tibial nerve SSR, an active electrode was placed on the plantar region, the reference electrode on the dorsal aspect of the foot, and the ground electrode on the pretibial region. The ipsilateral tibial nerve was stimulated. The electrical stimulation was applied to both wrists at the median nerves for each palm 


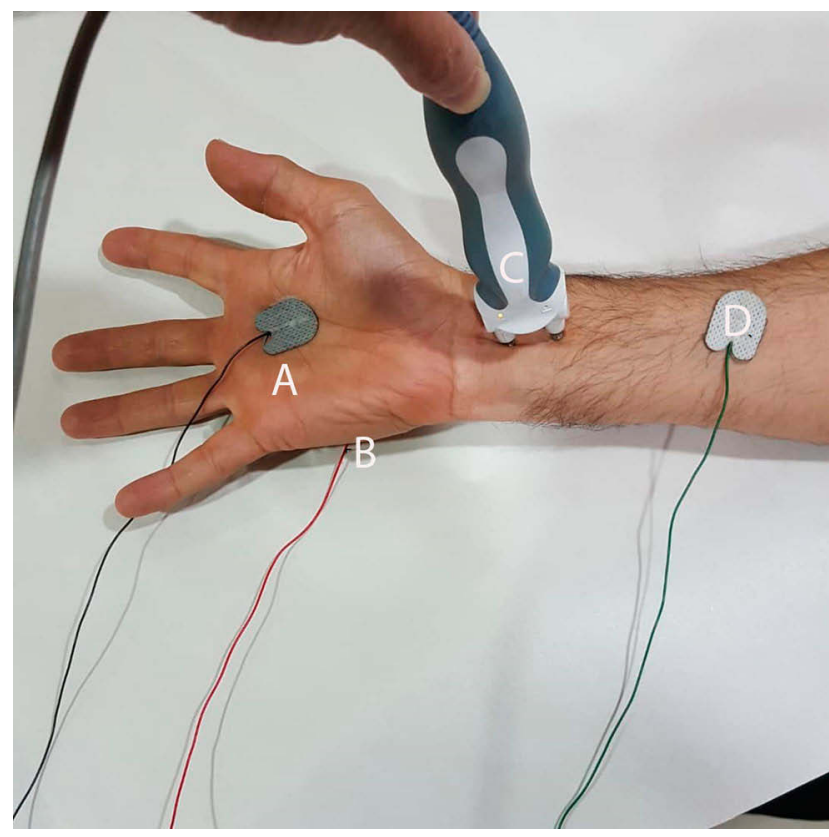

Figure I The sites of recording SSR from median nerve $(\mathbf{A})$ active electrode on the palm; (B) reference electrode on the dorsal aspect of the hand, (C) ground electrode on the forearm and (D) stimulate median nerve on the wrist. Abbreviation: SSR, sympathetic skin response.

recording site and both ankles at the tibial nerves for each recording site. (Figures 2 and 3)

The bandpass was $0.1-1000 \mathrm{~Hz}$, sensitivity $100-1000$ $\mathrm{H} / \mathrm{div}$, and the sweep speed $500 \mathrm{~ms} / \mathrm{div}$. The duration of the stimulus was between 0.1 and $0.2 \mathrm{~ms}$, and the stimulus intensity ranged from 10 to $40 \mathrm{~mA}$. Five responses were recorded, and the average of the potentials was taken into consideration. To avoid any habituation effect, stimulation was made with randomized intervals of varying intensity. Latency was measured from the onset of the stimulus artifact to the first deflection of the potential from baseline. Amplitude was measured from peak to peak. ${ }^{9,10}$

Only reproducible responses without any movement artifact that were consistent were selected for analysis (because each response can vary somewhat, only the consistent responses, with some variation in each subject, were selected for analysis). ${ }^{11}$

\section{Statistical Analyses}

The normality of data was tested using KolmogorovSmirnov and Shapiro-Wilk test; when the data were nonnormally distributed, a nonparametric test was used. The data were expressed as Mean $\pm \mathrm{SD}$, Inter-Quartile Range (IQR), and Confidence Interval for mean difference (CI). According to the parametrical or the non-parametrical distribution of the data, Independent Samples $t$-test or Mann Whitney test were

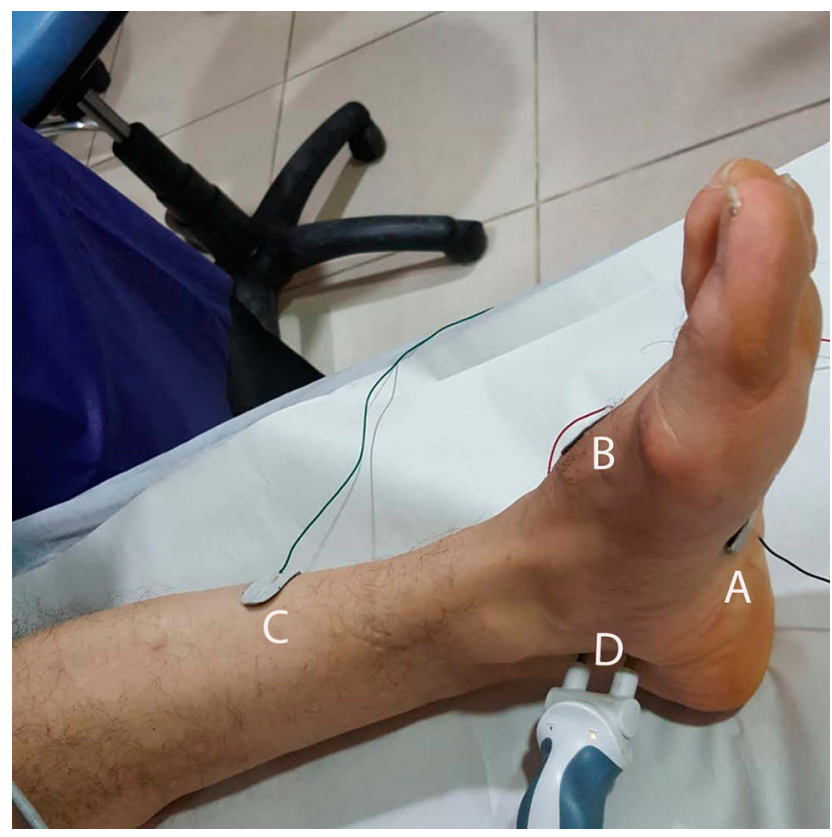

Figure 2 The sites of recording SSR from tibial nerve. (A) active electrode on the sole; (B) reference electrode on the dorsal aspect of the foot, $(\mathbf{C})$ ground electrode on the leg and (D) stimulate tibial nerve on the posterior to the medial malleolus. Abbreviation: SSR, sympathetic skin response.

used to compare the group differences. The collected data were analyzed using SPSS version 20. A P-value of less than 0.05 was considered statistically significant.

\section{Result}

The mean age and weight of men with CP type IIIb were similar to those of the control groups. There was no significant difference between the two groups in ethnicity distribution, marital status, and education level. The groups were different in terms of the current employment status ( $\mathrm{p}=$ $0.003)$, and about $5(30.7 \%)$ subjects in the CP type IIIb group were unemployed due to severe pain. Men with CP

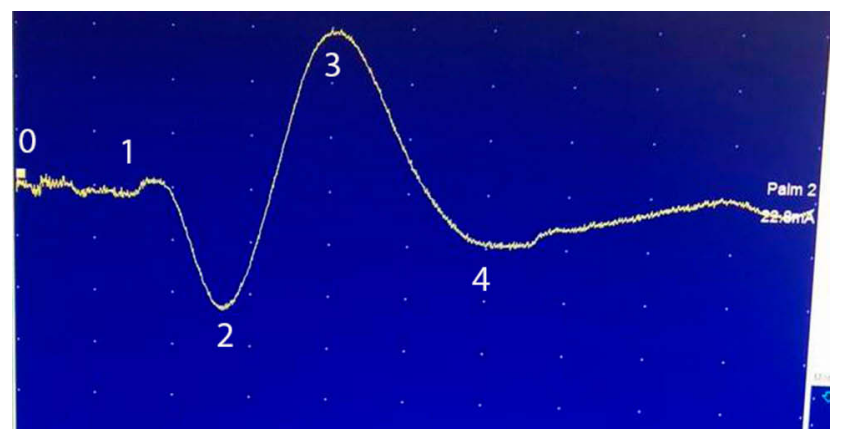

Figure 3 The SSR response wav form. 0-I: Horizontal line: onset latency(s). 2-3: Vertical line: peak to peak Amplitude $(\mu \mathrm{V})$. I-4: Horizontal line: total duration of SSR response(s).

Abbreviation: SSR, sympathetic skin response. 
Table I Demographic Characteristics of the Patient with Chronic Prostatitis Type Illb and Control Group

\begin{tabular}{|c|c|c|c|c|c|}
\hline \multirow[t]{2}{*}{ Variable } & \multirow[t]{2}{*}{ Subgroup Variable } & \multirow{2}{*}{$\begin{array}{l}\text { Total } \\
\text { Mean } \pm \text { SD \& N (\%) }\end{array}$} & \multirow{2}{*}{$\begin{array}{l}\text { Patient Group } \\
\text { Mean } \pm \text { SD \& N (\%) }\end{array}$} & \multirow{2}{*}{$\begin{array}{l}\text { Control Group } \\
\text { Mean } \pm \text { SD \& N (\%) }\end{array}$} & \multirow[t]{2}{*}{ p-value } \\
\hline & & & & & \\
\hline Age (years) & - & $31.00 \pm 3.59$ & $31.71 \pm 3.70$ & $30.61 \pm 3.54$ & 0.363 \\
\hline Height (cm) & - & $180.02 \pm 5.27$ & $178.35 \pm 4.14$ & $180.92 \pm 5.66$ & 0.144 \\
\hline Weight (Kg) & - & $78.58 \pm|2.4|$ & $77.21 \pm 10.69$ & $79.32 \pm 13.38$ & 0.615 \\
\hline BMI & - & $24.27 \pm 4.00$ & $24.32 \pm 3.78$ & $24.25 \pm 4.18$ & 0.959 \\
\hline \multirow[t]{4}{*}{ NIH-CPSI score* } & Pain score & $6.00 \pm 7.07$ & $15.42 \pm 1.22$ & $0.92 \pm 0.84$ & $<0.001$ \\
\hline & Urinary symptoms score & $3.27 \pm 3.02$ & $7.21 \pm 0.89$ & $1.15 \pm 0.73$ & $<0.001$ \\
\hline & QOL & $3.60 \pm 3.64$ & $8.28 \pm 1.20$ & $1.07 \pm 1.01$ & $<0.001$ \\
\hline & Total NIH score & $12.92 \pm 13.59$ & $30.92 \pm 3.23$ & $2.61 \pm 2.33$ & $<0.001$ \\
\hline \multirow[t]{2}{*}{ Educational levee } & Graduate & $12(30.0)$ & $4(28.6)$ & $8(30.8)$ & \multirow[t]{2}{*}{0.885} \\
\hline & High school & $28(70.0)$ & $10(71.4)$ & $18(69.2)$ & \\
\hline \multirow[t]{2}{*}{ Marital status } & Married & $31(77.5)$ & II (78.6) & $20(76.9)$ & \multirow[t]{2}{*}{0.905} \\
\hline & Single & $9(22.5)$ & $3(21.4)$ & $6(23.1)$ & \\
\hline \multirow[t]{2}{*}{ Employment status } & Employer & $35(87.5)$ & $9(64.3)$ & $26(100)$ & \multirow[t]{2}{*}{0.003} \\
\hline & Nonemployer & $5(12.5)$ & $5(35.7)$ & $0(0)$ & \\
\hline
\end{tabular}

Notes: P-values of $<0.05$ were considered significant. *The NIH-CPSI has a total score range from 0 to 43 , and it includes three subscales addressing pain (score range $0-2 \mathrm{I}$ ), urinary symptoms (score range $0-10$ ), and QOL (score range $0-12$ ).

Abbreviations: BMI, body mass index; NIH-CPSI, National Institute of Health Chronic Prostatitis Symptom Index; QOL, quality of life; SD, standard deviation.

type IIIb had significantly higher NIHCPSI scores (urinary symptoms score, pain score, quality of life, and Total NIH score) than those of the control group $(\mathrm{p}<0.001)$ (Table 1).

\section{Sympathetic Skin Responses}

Our result showed that there was no significant difference between the control group and patients with CP type IIIb group in terms of the latency in the left and right tibial nerve SSR, while the SSR latency in the left and right median nerve was significantly prolonged in patients with CP type IIIb, p= 0.039 and 0.006 , respectively. (Table 2)

Additionally, our result showed that there was no significant difference between the control group and patients with CP type IIIb in terms of the amplitude in the left tibial, left, and right median nerve, while amplitude was significantly reduced in the patients with CP type IIIb compared to the control group in right tibial nerve $(\mathrm{P}=0.017)$. (Table 3)

\section{Discussion}

Many patients who have CP syndrome visit the urologic outpatient department. They often present severe diagnostic and therapeutic problems and suffer from a debilitating disease. ${ }^{12}$ There is still no agreement on the criteria to be used for the diagnosis of $\mathrm{CP}$ syndrome. No guidelines have been published yet to provide the clinician with an approved diagnostic work-up plan. Our diagnostic efforts should be directed not only to establish the diagnosis of CP syndrome in general terms but also to identify an etiologic agent, if any, to detect the signs of inflammation and classify the patient into one of the four CP syndrome categories. ${ }^{12,13}$

A previously published article suggested that $\mathrm{CP} / \mathrm{CPPS}$ might be the result of peripheral neurogenic inflammation regulated by unmyelinated $\mathrm{C}$ fibers. These nerves are sensory neurons that have a characteristic "afferent/efferent" double function. ${ }^{14,15}$ Additionally, patients with CP type IIIb may experience sudomotor disturbances, such as hyperhidrosis or hypohidrosis. ${ }^{16,17}$

The role of the sympathetic nervous system in the complex regional pain syndrome (CRS) pathophysiology can be understood by the characteristic clinical signs of autonomic dysfunction, including edema, skin temperature and color fluctuations, and hyperhidrosis. Sympathetic dysfunction is considered the main pathophysiological mechanism of CRS. ${ }^{18,19}$ The SSR was used to measure electrical activity changes occurring in the sweat glands following sympathetic stimulation. Following an emotional or stress stimulus, changes in the sudomotor activity mediated by the sympathetic nervous system alter the skin's resistance to an electrical current. ${ }^{17}$

According to the present study, it can be concluded that the mean value of latency in patients with CP type IIIb is 
Table 2 Sympathetic Skin Response to Ipsilateral Stimulus Recorded from the Median and Tibial Nerves of the Participants in Terms of the Latency

\begin{tabular}{|l|l|l|l|l|l|l|l|}
\hline \multirow{2}{*}{ Variable } & \multicolumn{2}{l|}{ Patient Group } & \multicolumn{2}{l|}{ Control Group } & \multirow{2}{*}{ MD $\%$ Cl } & \multirow{2}{*}{ p-value } \\
\cline { 2 - 5 } & Mean (SD) & Median (IQR) & Mean (SD) & Median (IQR) & & & \\
\hline Right Median Latency (s) & $1.96(0.31)$ & $1.92(1.70-2.23)$ & $1.77(0.37)$ & $1.71(1.53-2.04)$ & 0.18 & 0.05 to 0.32 & 0.006 \\
Left Median Latency (s) & $1.91(0.31)$ & $1.89(1.70-2.07)$ & $1.78(0.35)$ & $1.71(1.56-2.02)$ & 0.13 & 0.006 to 0.26 & 0.039 \\
Right Tibial Latency (s) & $2.27(0.43)$ & $2.12(2.01-2.5)$ & $2.19(0.38)$ & $2.17(1.94-2.42)$ & 0.08 & -0.07 to 0.23 & 0.296 \\
Left Tibial Latency (s) & $2.13(0.45)$ & $2.10(1.84-2.37)$ & $2.17(0.41)$ & $2.22(2.03-2.40)$ & -0.04 & -0.20 to 0.11 & 0.588 \\
\hline
\end{tabular}

Note: P-values of $<0.05$ were considered significant.

Abbreviations: $\mathrm{Cl}$, confidence interval for mean difference; IQR, inter-quartile range; MD, mean difference between patients' group and control group; S, second; SD, standard deviation.

Table 3 Sympathetic Skin Response to Ipsilateral Stimulus Recorded from the Median and Tibial Nerves of the Participants in Terms of the Amplitude

\begin{tabular}{|l|l|l|l|l|l|l|l|}
\hline \multirow{2}{*}{ Variable } & \multicolumn{2}{|l|}{ Patient Group } & \multicolumn{2}{l|}{ Control Group } & \multirow{2}{*}{ MD } & \multirow{2}{*}{ 95\% CI } \\
\cline { 2 - 6 } & Mean (SD) & Median (IQR) & Mean (SD) & Median (IQR) & & \\
\hline Right Median Amplitude $(\mu \mathrm{V})$ & $1384.0(1375.5)$ & $1081.6(537.92-1838.3)$ & $1523.5(1080.5)$ & $1265.0(727.0-1889.1)$ & -139.5 & -590.9 to 312.0 & 0.542 \\
Left Median Amplitude $(\mu \mathrm{V})$ & $1388.6(1006.5)$ & $1042.5(466.2-2405.8)$ & $1620.0(1358.4)$ & $1277.5(866.6-1892.5)$ & -231.3 & -704.1 to 241.4 & 0.334 \\
Right Tibial Amplitude $(\mu \mathrm{V})$ & $867.2(1360.5)$ & $538.3(312.1-1088.3)$ & $1462.5(1237.0)$ & $1087.5(610.8-1865.0)$ & -595.2 & -1080.8 to -109.6 & 0.017 \\
Left Tibial Amplitude $(\mu \mathrm{V})$ & $1063.7(1121.4)$ & $740.8(369.5-1377.0)$ & $1248.4(1005.7)$ & $708.3(448.5-1942.1)$ & -184 & -581.6 to 212.0 & 0.359 \\
\hline
\end{tabular}

Note: P-values of $<0.05$ were considered significant.

Abbreviations: $\mathrm{Cl}$, confidence interval for mean difference; IQR, inter-quartile range; MD, mean difference between patients' group and control group; $\mu \mathrm{V}$, microvolt; SD, standard deviation.

higher than that of the control group in the left and right median region. In our study, the similarity of foot SSR latencies (right and left tibial latency) in men with CP type IIIb to those of the controls suggests the absence of significant differences of skin innervation between the groups. Although our aim was that there are SSR differences between the groups, the study outcome is not surprising because the painful area and urinary symptoms do not include any of the extremities; this is consistent with the results of Yilmaz et al. ${ }^{4}$

Moreover, we showed that the mean right tibial SSR amplitude was lower than that of the left foot, and the difference was significant in men with CP type IIIb. Although the amplitude is not universally considered a valid measure of normality versus abnormality, ${ }^{20}$ the SSR amplitude difference in the right tibial nerve in the CP type IIIb group may reflect the increased reaction to the stimulus at the right tibial nerve. This significant amplitude reduction can be a clue to the involvement of the sympathetic nervous system or effect of injury to the sensory pathways of the SSR. ${ }^{11}$

Other studies have focused on the significance of SSR to diagnose various diseases. As an example, Yilmaz et al evaluated the ANS changes in men with chronic pelvic pain syndrome (CPPS) and concluded that SSR findings were not conclusive and the only statistically significant finding was from the subjects with CPPS, in whom the mean lefthand SSR amplitude was higher than that of the right hand. Our results are not in the same line with those of the mentioned study. ${ }^{4}$ Roshanzamir et al found that dysfunction of SSR was associated with reduced bone mineral content; they found that SSR latency was significantly prolonged in electrical burn patients in comparison to the control group, and SSR amplitude was significantly reduced in these patients compared with the control group, which is consistent with the results of the present research, showing that SSR could be helpful as a diagnostic method. ${ }^{21}$

Pankaj and colleagues reported the diagnostic use of SSR that on 60 patients with CRPS affecting the hand. Patients were considered abnormal when there was an absent response or a response with a peak to- peak amplitude more than $50 \%$ compared to the contralateral hand. ${ }^{22}$ Mondelli et al evaluated the SSR in primary Raynaud's phenomenon and found that the latency of SSR was prolonged in patients with primary Raynaud's phenomenon. ${ }^{23}$

Another study reported that the SSR amplitude varied between people and that several factors influenced the SSR amplitude and SSR latencies remained consistent across 
significantly different recording sites, but not among different stimulation sites; therefore, measuring the SSR latency is essential. ${ }^{24}$ We evaluated the latency of SSR for the patients with CP type IIIb which was higher than control group.

Kim et al evaluated the predictive value of SSR in diagnosing complex regional pain syndrome (CRPS) and reported that five out of 6 CRPS patients showed prolonged latency on SSR (83\% sensitivity). While SSR latencies within normal limit were noted in 4 of 7 nonCRPS patients (57\% specificity), it was concluded that SSR might help detect CRPS, which is consistent with the present research. ${ }^{17}$

Selçuk et al evaluated the SSR in hemiplegic patients with and without CRPS, and SSR was recorded on the paretic and healthy hands after stimulation of the ipsilateral median nerve; the SSR amplitudes were increased at the hemiplegic limbs in patients affected by CRPS compared to individuals unaffected. They mentioned that SSR might be used in the evaluation of the sympathetic function in hemiplegic patients and in diagnosing of CRPS and monitoring its treatment. ${ }^{25}$

The current study had some limitations. One of them was the sample size of our study that was admittedly too small to run any robust statistical analysis $(n=14)$. This was just an observational report, which requires validation with a large sample size with randomized criteria. Moreover, the effect of SSR in men with CP type IIIb might be time-dependent and must be investigated in long-term studies. Additionally, our intent for this manuscript was not to draw the diagnosis of CP type IIIb from the SSR measurements or assign causality to the findings. We aimed to investigate any evidence that strengthens our overarching hypothesis that the ANS and $\mathrm{CP}$ type IIIb are intricately interrelated.

\section{Conclusion}

Sympathetic skin response is a low cost, a non-invasive method with a low possibility of complication, and might be a helpful diagnostic test for men with chronic prostatitis type IIIb. However, this observation needs to be validated in a large sample cohort study with long-term follow-up.

\section{Ethics Statement}

The Ethics Committee of Shiraz University of Medical Sciences approved the protocol of the study (IR.sums.med. rec.1397.431). The current study was performed according to the Institutional Committee for the Protection of Human Subjects, which was adopted by the 18th World Medical Assembly, Helsinki, Finland, and its later amendments.
Written informed consent was obtained from the patients. The present article was extracted from the thesis written by Azar Hosseini with ID number 17229, which was supported by Shiraz University of Medical Sciences.

\section{Acknowledgments}

The authors would like to thank Shiraz University of Medical Sciences, Shiraz, Iran, and also Center for Development of Clinical Research of Nemazee Hospital and Dr. Nasrin Shokrpour for editorial assistance.

\section{Author Contributions}

All authors contributed to data analysis, drafting or revising the article, gave final approval of the version to be published, and agreed to be accountable for all aspects of the work.

\section{Disclosure}

The authors declare that they have no competing interests.

\section{References}

1. Turini D, Beneforti P, Spinelli M, Malagutti S, Lazzeri M. Heat/ burning sensation induced by topical application of capsaicin on perineal cutaneous area: new approach in diagnosis and treatment of chronic prostatitis/chronic pelvic pain syndrome? Urology. 2006;67:910-913. doi:10.1016/j.urology.2005.11.028

2. Benelli A, Hossain H, Pilatz A, Weidner W. Prostatitis and its management. Eur Urol. 2017;16:132-137. doi:10.1016/j.eursup. 2016.03.001

3. Alexander RB, Propert KJ, Schaeffer AJ, et al. Ciprofloxacin or tamsulosin in men with chronic prostatitis/chronic pelvic pain syndrome: a randomized, double-blind trial. Ann Intern Med. 2004;141:581-589. doi:10.7326/0003-4819-141-8-200410190-00005

4. Yilmaz U, Liu Y-W, Berger RE, Yang CC. Autonomic nervous system changes in men with chronic pelvic pain syndrome. J Urol. 2007;177:2170-2174. doi:10.1016/j.juro.2007.01.144

5. Rommel O, Tegenthoff M, Pern U, Strumpf M, Zenz M, Malin J. Sympathetic skin response in patients with reflex sympathetic dystrophy. Clin Auton Res. 1995;5:205-210. doi:10.1007/BF01 824008

6. Lo Y, Leoh T, Loh L, Tan C. Statin therapy and small fibre neuropathy: a serial electrophysiological study. $J$ Neurol Sci. 2003;208:105-108. doi:10.1016/S0022-510X(02)00396-9

7. Claus D, Schondorf R. Sympathetic Skin Response. Recommendations for the Practice of Clinical Neurophysiology: Guidelines of the International Federation of Clinical Neurophysiology. Amsterdam: Elsevier; 1999:277-282.

8. Clemens JQ, Calhoun EA, Litwin MS, et al. Rescoring the NIH chronic prostatitis symptom index: nothing new. Prostate Cancer Prostatic Dis. 2009;12:285-287. doi:10.1038/pcan.2009.22

9. Farpour HR, Hajihosseini A, Moghimi Sarani E. Sympathetic skin response in patients with major depressive disorders. Armaghane Danesh. 2019;241087-1098.

10. Dumitru D, Zwarts MJ. Special Nerve Conduction Techniques. Electro Diagnostic Medicine. Philadelphia, PA: Hanley \& Belfus; 2002:249. 
11. Emad R, Zafarghasempour M, Roshanzamir S. Sympathetic skin response in incomplete spinal cord injury with urinary incontinence. Ann Indian Acad Neurol. 2013;16:234. doi:10.4103/0972-2327.112479

12. Pavone-Macaluso M. Chronic prostatitis syndrome: a common, but poorly understood condition. Part I. Eau-Ebu Update Series. 2007;5:1-15. doi:10.1016/j.eeus.2006.10.002

13. Pavone-Macaluso M. Chronic prostatitis syndrome: a common, but poorly understood condition. Part II. Eau-Ebu Update Series. 2007;5:16-25. doi:10.1016/j.eeus.2006.10.001

14. Miller LJ, Fischer KA, Goralnick SJ, et al. Nerve growth factor and chronic prostatitis/chronic pelvic pain syndrome. Uro. 2002;59:603-608. doi:10.1016/S0090-4295(01)01597-7

15. Maggi CA, Barbanti G, Santicioli P, et al. Cystometric evidence that capsaicin-sensitive nerves modulate the afferent branch of micturition reflex in humans. $J$ Urol. 1989;142:150-154. doi:10.1016/S00225347(17)38701-3

16. Gierthmühlen J, Maier C, Baron R, et al. Sensory signs in complex regional pain syndrome and peripheral nerve injury. $P A I N^{\mathbb{R}}$. 2012;153:765-774. doi:10.1016/j.pain.2011.11.009

17. Kim HJ, Yang HE, Kim DH, Park YG. Predictive value of sympathetic skin response in diagnosing complex regional pain syndrome: a case-control study. Ann Rehabil Med. 2015;39:116. doi:10.5535/ $\operatorname{arm} .2015 .39 .1 .116$

18. Bej MD, Schwartzman RJ. Abnormalities of cutaneous blood flow regulation in patients with reflex sympathetic dystrophy as measured by laser Doppler fluxmetry. Arch Neurol. 1991;48:912-915. doi:10.1001/ archneur.1991.00530210038020
19. Marinus J, Moseley GL, Birklein F, et al. Clinical features and pathophysiology of complex regional pain syndrome. Lancet Neurol. 2011;10:637-648. doi:10.1016/S1474-4422(11)70106-5

20. Vetrugno R, Liguori R, Cortelli P, Montagna P. Sympathetic skin response. Clin Auton Res. 2003;13:256-270. doi:10.1007/s10286003-0107-5

21. Roshanzamir S, Dabbaghmanesh MH, Dabbaghmanesh A, Nejati S. Autonomic dysfunction and osteoporosis after electrical burn. Burns. 2016;42:583-588. doi:10.1016/j.burns.2015.09.009

22. Pankaj A, Kotwal P, Mittal R, Deepak K, Bal C. Diagnosis of post-traumatic complex regional pain syndrome of the hand: current role of sympathetic skin response and three-phase bone scintigraphy. J Orthop Surg. 2006;14:284-290. doi:10.1177/230949900601400310

23. Mondelli M, de Stefano R, Rossi S, Aretini A, Romano C. Sympathetic skin response in primary Raynaud's phenomenon. Clin Auton Res. 2009;19:355-362. doi:10.1007/s10286-009-0021-6

24. Uncini A, Pullman S, Lovelace R, Gambi D. The sympathetic skin response: normal values, elucidation of afferent components and application limits. J Neurol Sci. 1988;87:299-306. doi:10.1016/ 0022-510X(88)90254-7

25. Selcuk B, Ersoz M, Inanir M, Kurtaran A, Akyuz M. Sympathetic skin responses in hemiplegic patients with and without complex regional pain syndrome. Neurol India. 2006;54:279. doi:10.4103/ 0028-3886.27153

\section{Publish your work in this journal}

Research and Reports in Urology is an international, peer-reviewed, open access journal publishing original research, reports, editorials, reviews and commentaries on all aspects of adult and pediatric urology in the clinic and laboratory including the following topics: Pathology, pathophysiology of urological disease; Investigation and treatment of urological disease; Pharmacology of drugs used for the treatment of urological disease. The manuscript management system is completely online and includes a very quick and fair peer-review system, which is all easy to use. Visit http://www.dovepress.com/ testimonials.php to read real quotes from published authors. 